Japan. J. Math.

Vol. 14, No. 1, 1988

\title{
On decaying entire positive solutions of semilinear elliptic equations
}

\author{
By Yasuhiro Furusho
}

(Received September 9, 1986)

\section{§1. Introduction}

This paper is concerned with the existence of positive entire solutions of semilinear equations of the form

$$
L u=\lambda f(x, u) \quad \text { in } \boldsymbol{R}^{N}, N \geqq 2,
$$

where $L$ denotes a uniformly elliptic differential operator with smooth real valued coefficients defined by

$$
L u=-\left(\sum_{i, j=1}^{N} a_{i j}(x) \frac{\partial^{2} u}{\partial x_{i} \partial x_{j}}+\sum_{i=1}^{N} b_{i}(x) \frac{\partial u}{\partial x_{i}}\right)+c(x) u,
$$

$f(x, u)$ is a locally Hölder continuous function in $\boldsymbol{R}^{N} \times \overline{\boldsymbol{R}}_{+}, \overline{\boldsymbol{R}}_{+}=[0, \infty)$, and $\lambda$ is a real constant.

By an entire solution of (1.1) we mean a function $u \in C^{2}\left(\boldsymbol{R}^{N}\right)$ which satisfies (1.1) throughout $\boldsymbol{R}^{N}$. Our purpose here is to give criteria for the existence of decaying entire positive solutions of (1.1), i.e., those entire solutions which are positive in $\boldsymbol{R}^{N}$ and have the uniform limit zero as $|x| \rightarrow \infty$, under suitable hypotheses on the nonlinearity of $f(x, u)$.

Our first theorem, Theorem 2.1 below, establishes the existence of a decaying entire positive solution $u(x)$ of (1.1) for sufficiently small $\lambda>0$ under the hypotheses that $f(x, u) \geqq 0$ and $\lim _{u \rightarrow 0+} f(x, u) / u=\infty$ uniformly in some bounded domain in $\boldsymbol{R}^{N}$. On the other hand, in Theorem 2.2 we show that there exists a maximal decaying entire positive solution of (1.1) in the case where $f(x, u)$ has bounded nonlinearity at $u=\infty$ (see hypothesis $\left(F_{4}\right)$ below); we also investigate the case where $\lim _{u \rightarrow 0+} f(x, u) / u$ is finite. As a byproduct of Theorem 2.2 we can prove the uniqueness of decaying entire positive solution of the equation

$$
-\Delta u+c(x) u=\lambda f(x, u) \quad \text { in } \boldsymbol{R}^{N}, N \geqq 3,
$$

when $f(x, u) / u$ is strictly decreasing in $u>0$ (Theorem 5.1). Our Theorems 2.1 
and 2.2 can be applied to the following mixed sublinear and superlinear equations

$$
-\Delta u+c(x) u=\lambda\left(\Phi(x) u^{\gamma}+\Psi(x) u^{\delta}\right) \quad \text { in } R^{N}, N \geqq 3
$$

and

$$
-\Delta u+c(x) u=\lambda\left(\Phi(x) u^{\gamma}-\Psi(x) u^{\delta}\right) \quad \text { in } R^{N}, N \geqq 3,
$$

where $0 \leqq \gamma<1 \leqq \delta, \gamma \neq \delta$, and $c, \Phi$ and $\Psi$ are nonnegative locally Hölder continuous functions in $\boldsymbol{R}^{N}$. For (1.4) the possibility of $\gamma=1$ is allowed. The statements of Theorems 2.1 and 2.2 are given in $\S 2$. The proofs of these theorems are presented in $\S 4$. Analytical tools which are needed in the proofs are provided in $\S 3$. Section 5 concerns specialization of the main results to equations (1.3) and (1.4).

The existence of entire positive solutions of (1.1) has been proved under various structure hypotheses for $L$ and $f(x, u)$ by many authors (see e.g. [3], [6], [7], [9], [11]-[19], [21]). Among them the results of Fukagai [6, 7], of Kusano and Trench [15] and of Noussair and Swanson [19] are closely related to this paper. Kusano and Trench [15, Theorem 2] gave sufficient conditions for (1.3) to have decaying entire positive solution in the case where $c(x) \equiv 0$, and $\Phi$ and $\Psi$ are radially symmetric functions. As far as we are aware, there is no result on the existence of decaying entire solutions for the general equation (1.1) except for a very recent result in [19], where systems of equations generalizing (1.1) are considered.

\section{§2. Statement of main theorems}

Throughout this paper we assume that the coefficients of $L$ of (1.2) satisfy $a_{i j}=a_{j i} \in C_{l o c}^{1+\alpha}\left(\boldsymbol{R}^{N}\right), b_{i}, c \in C_{l o c}^{\alpha}\left(\boldsymbol{R}^{N}\right), i, j=1, \cdots, N, 0<\alpha<1$ and $c(x) \geqq 0$ in $R^{N}$ and that $L$ is uniformly elliptic in $R^{N}$, i.e., there is a constant $a_{0}>0$ such that

$$
\sum_{i, j=1}^{N} a_{i j}(x) \xi_{i} \xi_{j} \geqq a_{0}|\xi|^{2} \quad \text { for } x, \xi \in R^{N},
$$

where $|\xi|$ denotes the Euclidean length of $\xi \in \boldsymbol{R}^{N}$.

The following notation is employed:

$$
\begin{aligned}
& \boldsymbol{R}_{+}=(0, \infty), \quad \overline{\boldsymbol{R}}_{+}=[0, \infty), \quad B_{R}=\left\{x \in R^{N}:|x|<R\right\} \quad \text { for } R>0, \\
& a(x)=\sum_{i, j=1}^{N} a_{i j}(x) x_{i} x_{j} /|x|^{2}, \\
& b(x)=\left(\sum_{i=1}^{N}\left(b_{i}(x) x_{i}+a_{i i}(x)\right)-a(x)\right) /|x|
\end{aligned}
$$


$b_{*}(r)=\min _{|x|=r} \frac{b(x)}{a(x)}$,

$p_{*}(r)=\exp \left(\int_{R}^{r} b_{*}(s) d s\right), \quad \pi_{*}(r)=\int_{r}^{\infty} \frac{d s}{p_{*}(s)}$ (if the integral converges)

$h^{*}(r)=\max _{|x|=r} \frac{h(x)}{a(x)} \quad$ for a continuous function $h$.

Making use of the above $a$ and $b$, we obtain an identity

$$
L u(r)=-a(x) u^{\prime \prime}(r)-b(x) u^{\prime}(r)+c(x) u(r), \quad r=|x|>0
$$

for a smooth function $u(r)$ defined in $R_{+}$, where ${ }^{\prime}=d / d r$.

In this paper, we make the following standing hypothesis $(H)$ for the operator $L$ :

$$
\int_{R}^{\infty} \frac{d s}{p_{*}(s)}<\infty
$$

so that the integral $\pi_{*}(r)$ exists for any $r>0$. It is easy to see that in the case where $L=-\Delta, \Delta$ being the $N$-dimensional Laplacian, hypothesis $(H)$ holds if and only if $N \geqq 3$. For other examples of $L$ satisfying $(H)$ we refer to [9] (see also Remark 4.1 below).

The conditions below will be imposed on the function $f(x, u)$ in (1.1).

$\left(F_{1}\right) \quad f(x, u) \in C_{l o c}^{\theta}\left(\boldsymbol{R}^{N} \times \overline{\boldsymbol{R}}_{+}\right), 0<\theta<1$. For any bounded domain $\Omega \subset \boldsymbol{R}^{N}$ and any constant $U>0$, there corresponds a positive constant $M$ such that $f(x, u)+M u$ is nondecreasing in $u \in[0, U]$ for any fixed $x \in \Omega$.

$\left(F_{2}\right)$ There exists a positive function $m \in C_{l o c}^{\alpha}\left(\boldsymbol{R}^{N}\right)$ such that for any $U>0$

$$
0 \leqq f(x, u) \leqq C_{U} m(x), \quad x \in R^{N}, \quad 0<u \leqq U,
$$

where $C_{U}$ is a positive constant depending only on $U$.

$\left(F_{3}\right) \lim _{u \rightarrow 0+}(f(x, u) / u)=+\infty$ uniformly on some bounded domain $\Omega_{0}$ in $\boldsymbol{R}^{N}$.

Condition $\left(F_{3}\right)$ is satisfied, for example, if $f(x, 0)>0$ in $R^{N}$.

EXAMPLE 2.1. The function

$$
f(x, u)=\Phi(x) u^{r}+\Psi(x) u^{\delta}, \quad 0 \leqq \gamma<1, \quad \delta \geqq \gamma
$$

satisfies $\left(F_{1}\right)-\left(F_{3}\right)$ provided $\Phi, \Psi \in C_{l o c}^{\alpha}\left(\boldsymbol{R}^{N}\right), \Phi(x)>0$ and $\Psi(x) \geqq 0$ in $\boldsymbol{R}^{N}$. The possibility of $\delta \geqq 1$ or $\Psi(x) \equiv 0$ in $R^{N}$ is allowed.

The first main theorem in this paper is the following.

Theorem 2.1. Assume that $(H),\left(F_{1}\right)-\left(F_{3}\right)$ are satisfied. If 


$$
\int_{R}^{\infty} \pi_{*}(r) p_{*}(r) m^{*}(r) d r<\infty,
$$

then there exists $\lambda^{*}>0$ such that, for every $\lambda \in\left(0, \lambda^{*}\right)$, (1.1) possesses an entire positive solution $u$ satisfying $\lim _{|x| \rightarrow \infty} u(x)=0$.

Before stating the next result, we prepare some notation and terminology. Let $\lambda^{*}$ and $\lambda_{*}$ denote, respectively, the least upper bound and the greatest lower bound of $\lambda>0$ for which (1.1) has an entire positive solution $u$ satisfying $\lim _{|x| \rightarrow \infty} u(x)=0$.

For a locally Hölder continuous positive function $\tilde{m}$ in $R^{N}$ and $n=1,2$, $\cdots$, let $\lambda_{n}(\tilde{m})$ be the principal eigenvalue of the Dirichlet problem

$$
L \varphi=\lambda \tilde{m}(x) \varphi \text { in } B_{n}, \quad \varphi=0 \text { on } \partial B_{n} .
$$

It is well known that $\lambda_{n}(\tilde{m})$ is positive by Krein-Rutman's theory ([1, Theorem 4.3]) and decreases in $n$ ( see e.g. [9, p. 176]). Let us denote the limit of $\lambda_{n}(\tilde{m})$ as $n \rightarrow \infty$ by $\lambda_{0}(\tilde{m}): \lambda_{0}(\tilde{m})=\lim _{n \rightarrow \infty} \lambda_{n}(\tilde{m})$.

An entire positive solution $\bar{u}$ of (1.1) is said to be a maximal decaying entire positive solution of (1.1) if $\lim _{|x| \rightarrow \infty} \bar{u}(x)=0$ and $0<u(x) \leqq \bar{u}(x)$ in $\boldsymbol{R}^{N}$ for any entire positive solution $u$ of (1.1) such that $\lim _{|x| \rightarrow \infty} u(x)=0$.

The following conditions on $f(x, u)$ are needed in establishing the existence of a maximal decaying entire positive solution of (1.1).

$\left(F_{4}\right)$ There exist a positive function $m \in C_{l o c}^{\alpha}\left(\boldsymbol{R}^{N}\right)$ and a nonnegative function $g \in C_{l o c}^{\alpha}\left(\boldsymbol{R}^{N}\right)$ such that, for any constant $\mu>0$,

$$
f(x, u) \leqq \mu m(x) u+C(\mu) g(x), \quad x \in \boldsymbol{R}^{N}, \quad u \in \overline{\boldsymbol{R}}_{+},
$$

where $C(\mu)$ is a positive constant depending only on $\mu>0$ but not on $u$.

$\left(F_{5}\right)$ There exists a positive function $\tilde{m} \in C_{l o c}^{\alpha}\left(\boldsymbol{R}^{N}\right)$ such that

$$
\lim _{u \rightarrow 0+} \frac{f(x, u)}{\tilde{m}(x) u}=1 \quad \text { uniformly in } R^{N} \text {. }
$$

EXAmple 2.2. Let $\Phi$ and $\Psi$ be as in Example 2.1. Then the function

$$
f(x, u)=\Phi(x) u^{\gamma}-\Psi(x) u^{\delta}, \quad 0 \leqq \gamma \leqq 1 \leqq \delta, \quad \gamma \neq \delta
$$

satisfies $\left(F_{4}\right)$ and either $\left(F_{3}\right)$ or $\left(F_{5}\right)$ provided $\Psi(x) / \Phi(x)$ is bounded above and below by some positive constants in $R^{N}$. In case $\gamma \neq 1, \Psi(x) \equiv 0$ is allowed.

Theorem 2.2. Assume that $(H)$ holds and f satisfies $\left(F_{1}\right),\left(F_{4}\right)$ and either $\left(F_{3}\right)$ or $\left(F_{5}\right)$. Suppose for $m$ and $g$ in $\left(F_{4}\right)$ that

$$
\int_{R}^{\infty} \pi_{*}(r) p_{*}(r)\left\{m^{*}(r)+g^{*}(r)\right\} d r<\infty
$$


for some $R>0$.

(i) If $f$ satisfies $\left(F_{3}\right)$, then $\lambda_{*}=0$ and $\lambda^{*}=\infty$.

(ii) If $f$ satisfies $\left(F_{5}\right)$, then $\lambda_{*} \leqq \lambda_{0}(\tilde{m})$ and $\lambda^{*}=\infty$. In particular, $\lambda_{*}$ is positive provided

$$
\sup _{x \in R^{N}, u>0} \frac{f(x, u)}{m(x) u}<\infty
$$

Furthermore, for every $\lambda>\lambda_{*}$, (1.1) possesses a maximal decaying entire positive solution $\bar{u}$ in both cases (i) and (ii) above.

\section{§ 3. Preliminaries}

Let $f(x, u)$ be a locally Hölder continuous function defined in $\boldsymbol{R}^{N} \times \boldsymbol{R}$. A continuous function $v$ in $\boldsymbol{R}^{N}$ is said to be a supersolution of

$$
L u=f(x, u) \quad \text { in } R^{N},
$$

if there exists a smooth bounded domain $G_{1}$ in $R^{N}$ such that $v_{j}=\left.v\right|_{\bar{G}_{j}}$ (the restriction of $v$ to $\bar{G}_{j}$, where $\left.G_{2}=R^{N} \backslash \bar{G}_{1}\right), j=1,2$, satisfy the conditions

$$
\begin{aligned}
& v_{j} \in C^{2}\left(G_{j}\right) \cap C^{1}\left(\bar{G}_{j}\right), \\
& L v_{j} \geqq f\left(x, v_{j}\right), \quad x \in G_{j}, \\
& \frac{\partial v_{1}}{\partial \nu} \geqq \frac{\partial v_{2}}{\partial \nu} \quad \text { on } \quad \partial G_{1},
\end{aligned}
$$

where $\nu$ is an outward (with respect to $G_{1}$ ) conormal vector to $\partial G_{1}$ associated with $L$.

A subsolution of (3.1) is defined by reversing the above inequality signs.

Lemma 3.1. Assume that $f \in C_{l o c}^{\theta}\left(\boldsymbol{R}^{N} \times R\right), 0<\theta<1$, and that, for any bounded domain $\Omega \subset \boldsymbol{R}^{N}$ and any finite interval $I \subset R$, there is a constant $M>0$ such that $f(x, u)+M u$ is nondecreasing in $u \in I$ for any fixed $x \in \Omega$. Then the existence of a supersolution $v$ and a subsolution $w$ of (3.1) with the property $w(x) \leqq v(x)$ in $\boldsymbol{R}^{N}$ implies the existence of a solution $\bar{u} \in C^{2}\left(\boldsymbol{R}^{N}\right)$ of (3.1) such that the inequalities

$$
w(x) \leqq u(x) \leqq \bar{u}(x) \leqq v(x), \quad x \in \boldsymbol{R}^{N}
$$

hold for any solution $u$ of (3.1) satisfying $w(x) \leqq u(x) \leqq v(x)$ in $\boldsymbol{R}^{N}$.

The proof of this lemma is the same as that of [9, Lemma 4.2], and will be omitted.

The next lemma is an analogue of Lemma 5.3 of [8] concerning the exterior boundary value problems for $L u=\lambda f(x, u)$. 
LEMMA 3.2. Let $m$ be a continuous function in $\boldsymbol{R}^{N}$ and $\lambda$ be a constant. Assume that there is a function $V \in C^{2}\left(\boldsymbol{R}^{N}\right)$ satisfying

$$
L V(x) \geqq \lambda m(x) V(x), \quad V(x)>0, \quad x \in \boldsymbol{R}^{N} .
$$

If $u \in C^{2}\left(\boldsymbol{R}^{N}\right)$ satisfies

$$
L u(x) \geqq \lambda m(x) u(x), \quad x \in R^{N}
$$

and

$$
\liminf _{|x| \rightarrow \infty} \frac{u(x)}{V(x)} \geqq 0
$$

then $u(x)>0$ in $R^{N}$ unless $u(x) \equiv 0$.

Proof. We first prove the nonnegativity of $u$. Fix an $\varepsilon>0$ and choose an $R>0$ so that

$$
\frac{u(x)}{V(x)} \geqq-\varepsilon, \quad|x| \geqq R .
$$

Then, by (3.2), (3.3) and (3.5) the function $\tilde{u}(x)=u(x)+\varepsilon V(x)$ satisfies

$$
L \tilde{u}(x)-\lambda m(x) \tilde{u}(x) \geqq 0 \quad \text { in } \quad B_{R}, \quad \tilde{u}(x) \geqq 0 \quad \text { on } \quad \partial B_{R} .
$$

Hence, $\tilde{u}(x) / V(x) \geqq 0$ in $\boldsymbol{R}^{N}$ by the generalized maximum principle ([20, p. 73 , Theorem 10]). Combining this with (3.5), we have

$$
\tilde{u}(x)=u(x)+\varepsilon V(x) \geqq 0 \quad \text { in } \boldsymbol{R}^{N} .
$$

Since $\varepsilon>0$ is arbitrary, we conclude that $u(x) \geqq 0$ in $\boldsymbol{R}^{N}$.

Next, suppose that $u(x) \geqq 0$ and $u(x) \neq 0$. Then, by (3.3) and the generalized maximum principle mentioned above the function $u(x) / V(x)$ cannot attain a nonpositive minimum in $\boldsymbol{R}^{N}$. This implies $u(x) / V(x)>0$ and so $u(x)$ $>0$ in $\boldsymbol{R}^{N}$.

The next lemma concerns the following initial value problem for an ordinary differential equation

$$
\begin{aligned}
& \left(p(r) y^{\prime}\right)^{\prime}+\lambda q(r) y+h(r)=0, \quad r>R, \\
& y(R)=\xi, \quad y^{\prime}(R)=-\eta,
\end{aligned}
$$

where ${ }^{\prime}=d / d r, \lambda \in R$, and the functions $p, q$ and $h$ satisfy the conditions:

$$
\begin{aligned}
& p \in C^{1}([R, \infty)) \text { and } p(r)>0 \quad \text { in }[R, \infty), \quad q, h \in C([R, \infty)) \text { and } \\
& q(r) \geqq 0, \quad h(r) \geqq 0 \text { and } q(r) \not \equiv 0, \quad h(r) \not \equiv 0 \text { in }[R, \infty) .
\end{aligned}
$$


LEMMA 3.3. Assume that $\pi(r) \equiv \int_{r}^{\infty} d s / p(s)<\infty$ for $r \geqq R$ and

$$
\int_{R}^{\infty} \pi(r)\{q(r)+h(r)\} d r<\infty
$$

and let $0 \leqq \lambda<\left(\int_{R}^{\infty} \pi(r) q(r) d r\right)^{-1}$. If $\xi_{0}>0$ is sufficiently large, then for every $\xi>\xi_{0}$, there corresponds a constant $\eta=\eta(\xi)>0$ such that the solution $y$ of (3.6) satisfies

$$
y(r)>0, \quad y^{\prime}(r)<0, \quad r \in[R, \infty) \quad \text { and } \quad \lim _{r \rightarrow \infty} y(r)=0 .
$$

Proof. Put $\kappa=\int_{R}^{\infty} \pi(r) q(r) d r$ and let $0 \leqq \lambda<\kappa^{-1}$. We begin by proving that the solution $z_{0}$ of the initial value problem

$$
\begin{aligned}
& \left(p(r) z^{\prime}\right)^{\prime}+\lambda q(r) z=0, \quad r>R, \\
& z(R)=0, \quad z^{\prime}(R)=1
\end{aligned}
$$

is bounded and positive in $(R, \infty)$. In fact, by the change of variables

$$
s=\frac{1}{\pi(r)}, \quad r \geqq R, \quad \text { and } \quad Z(s)=\frac{z(r(s))}{\pi(r(s))}, \quad s \geqq S=\frac{1}{\pi(R)},
$$

(3.8) becomes

$$
\frac{d^{2} Z}{d s^{2}}+\lambda\left(p \pi^{4} q\right)(r(s)) Z=0, \quad s>S
$$

Since

$$
\int_{S}^{\infty} s\left(p \pi^{4} q\right)(r(s)) d s=\int_{R}^{\infty} \pi(r) q(r) d r<\infty,
$$

(3.10) has a pair of solutions $Z_{1}$ and $Z_{2}$ such that

$$
\lim _{s \rightarrow \infty} \frac{Z_{1}(s)}{s}=1 \text { and } \lim _{s \rightarrow \infty} Z_{2}(s)=1
$$

([10, p. 428, Theorem 9.1.1]). Consequently, (3.8) has a pair of soultions $z_{1}$ and $z_{2}$ such that $\lim _{r \rightarrow \infty} z_{1}(r)=1$ and $\lim _{r \rightarrow \infty} z_{2}(r)=0$, which implies the boundedness of $z_{0}$.

To prove the positivity of $z_{0}$, suppose that $z_{0}$ has a zero in $(R, \infty)$. Then, there are $R_{1}>R$ and $\tilde{R} \in\left(R, R_{1}\right)$ such that

$$
z_{0}(r)>0, \quad R<r<R_{1}, \quad z_{0}\left(R_{1}\right)=0 \quad \text { and } \quad z_{0}^{\prime}(\tilde{R})=0 .
$$


In view of the representation for $z_{0}$

$$
z_{0}(r)=z_{0}(\tilde{R})-\lambda \int_{\tilde{R}}^{r}(\pi(t)-\pi(r)) q(t) z_{0}(t) d t
$$

which follows from (3.8), we have

$$
\begin{aligned}
z_{0}\left(R_{1}\right) & =z_{0}(\tilde{R})-\lambda \int_{\tilde{R}}^{R_{1}}\left(\pi(t)-\pi\left(R_{1}\right)\right) q(t) z_{0}(t) d t \\
& \geqq z_{0}(\tilde{R})\left(1-\lambda \int_{\tilde{R}}^{R_{1}} \pi(t) q(t) d t\right) \geqq z_{0}(\tilde{R})(1-\lambda \kappa)>0,
\end{aligned}
$$

where we have used the fact that $z_{0}^{\prime}(r) \leqq 0$ for $r \in\left(\tilde{R}, R_{1}\right)$. This contradiction shows that $z_{0}(r)>0$ throughout $(R, \infty)$.

Now, let us prove the assertion of the lemma. Put

$$
\sigma=\int_{R}^{\infty} \pi(r) h(r) d r \quad \text { and } \quad \xi_{0}=\sigma(1-\lambda \kappa)^{-1} \text { for } 0 \leqq \lambda<\kappa^{-1} .
$$

For a fixed $\xi>\xi_{0}$ we denote the unique solution of (3.6) by $y(r ; \eta)$. Integrating (3.6) twice from $R$ to $r$, we have

$$
\begin{aligned}
y(r ; \eta)= & \xi-p(R)(\pi(R)-\pi(r)) \eta-\lambda \int_{R}^{r}(\pi(t)-\pi(r)) q(t) y(t ; \eta) d t \\
& -\int_{R}^{r}(\pi(t)-\pi(r)) h(t) d t, \quad r \geqq R,
\end{aligned}
$$

from which it follows that $y(r ; \eta)>0$ for $r>R$ if $\eta<\{\xi(1-\lambda k)-\sigma\} /(p(R) \pi(R))$ and that $y(\tilde{r} ; \eta)=0$ for some $\tilde{r}>R$ if $\eta>\xi /(p(R) \pi(R))$. The first fact is shown analogously to the positivity of $z_{0}$. To see the second fact it suffices to notice that if $y(r ; \eta)>0$ for all $r>R$, then we have $y(r ; \eta) \leqq \xi-p(R) \pi(R) \eta+p(R) \pi(r) \eta$, $r>R$, the right side of which tends to $\xi-p(R) \pi(R) \eta<0$ as $r \rightarrow \infty$ provided $\eta>$ $\xi /(p(R) \pi(R))$. Now, consider the set $A=\{\eta>0: y(\tilde{r} ; \eta)=0$ for some $\tilde{r}>R\}$. Then, we see that $A$ is open in $\boldsymbol{R}_{+}$by the continuous dependence of solutions of (3.6) on initial data and that

$$
\eta^{\prime} \in A \text { if } \eta^{\prime}>\eta^{\prime \prime} \quad \text { and } \quad \eta^{\prime \prime} \in A
$$

from the relation $y\left(r ; \eta^{\prime \prime}\right)-y\left(r ; \eta^{\prime}\right)=\left(\eta^{\prime}-\eta^{\prime \prime}\right) z_{0}(r)>0$ in $(R, \infty)$ for $\eta^{\prime}>\eta^{\prime \prime}$. For $\xi>\xi_{0}$ we define $\eta(\xi)=\inf \{\eta: \eta \in A\}$. Then, $\left.\eta(\xi) \geqq\{\xi(1-\lambda \kappa)-\sigma)\right\} /(p(R) \pi(R))$ and the following hold:

$$
\begin{gathered}
\eta \in A \quad \text { for } \eta>\eta(\xi), \\
y(r ; \eta(\xi))>0 \text { and } y^{\prime}(r ; \eta(\xi))<0, \quad r>R, \\
\lim _{r \rightarrow \infty} y(r ; \eta(\xi))=0 .
\end{gathered}
$$


In fact, (3.13) follows from (3.12). The positivity of $y(r ; \eta(\xi))$ follows from the definition of $\eta(\xi)$ and the fact that $A$ is open. Differentiating (3.11) and using the positivity of $y(r ; \eta(\xi))$, we get the second relation in (3.14). To prove (3.15), assume that $l_{0}=\lim _{r \rightarrow \infty} y(r ; \eta(\xi))>0$. Putting $\varepsilon=l_{0} /\left(2 \sup _{r \geqq R} z_{0}(r)\right)>0$, we obtain

$$
\begin{aligned}
y(r ; \eta) & =y(r ; \eta(\xi))-(y(r ; \eta(\xi))-y(r ; \eta)) \geqq l_{0}-(\eta-\eta(\xi)) z_{0}(r) \\
& \geqq l_{0}-\varepsilon z_{0}(r) \geqq \frac{1}{2} l_{0}>0 \quad \text { in }(R, \infty)
\end{aligned}
$$

provided $\eta(\xi)<\eta<\eta(\xi)+\varepsilon$. This shows that $\eta \notin A$ for any $\eta \in(\eta(\xi), \eta(\xi)+\varepsilon)$ which contradicts (3.13). Therefore (3.15) holds. This finishes the proof.

From the above proof we have the following.

REMARK 3.1. For $\lambda \in\left[0, \kappa^{-1}\right), \xi_{0}$ in Lemma 3.3 can be taken to be $\xi_{0}=$ $\sigma /(1-\lambda k)$. Furthermore, if $0<\lambda<\hat{\lambda}<\kappa^{-1}$, we have $\eta(\xi) \geqq\{\xi(1-\hat{\lambda} k)-\sigma\} /(p(R) \pi(R))$ for $\xi>\xi_{0}$.

The following lemma, which is used in the proofs of Theorems 2.1 and 2.2, improves Theorem 1 of Friedman [5] and Theorem 1 of Noussair and Swanson [18].

Lemma 3.4. Let $m$ and $g$ be locally Hölder continuous functions in $\boldsymbol{R}^{N}$ with exponent $\alpha, 0<\alpha<1$, and assume that $m(x)>0$ and $g(x) \geqq 0, \not \equiv 0$ in $R^{N}$. If

$$
\int_{R}^{\infty} \pi_{*}(r) p_{*}(r)\left\{m^{*}(r)+g^{*}(r)\right\} d r<\infty
$$

for some $R>0$, then there is a positive constant $\tilde{\lambda}$ such that, for every $\lambda \in[0, \tilde{\lambda})$, the linear equation

$$
L u=\lambda m(x) u+g(x) \quad \text { in } R^{N}
$$

possesses a unique positive solution $u \in C_{l o c}^{2+\alpha}\left(\boldsymbol{R}^{N}\right)$ satisfying $\lim _{|x| \rightarrow \infty} u(x)=0$.

Proof. We first note that by [9, Theorem 4.3] one can choose a positive constant $\lambda_{1}>0$ such that, for every $\lambda \in\left[0, \lambda_{1}\right]$, there exists a function $V \in$ $C_{l o c}^{2+\alpha}\left(\boldsymbol{R}^{N}\right)$ satisfying

$$
L V \geqq \lambda m(x) V, \quad 0<C_{1} \leqq V(x) \leqq C_{2}<\infty \quad \text { in } \quad R^{N},
$$

for some constants $C_{1}$ and $C_{2}$.

Since $m(x)+g(x)>0$ in $\bar{B}_{R}$, there is a constant $\lambda_{2}>0$ such that the Dirichlet problem 


$$
\begin{array}{rr}
-\left(\sum_{i, j=1}^{N} a_{i j}(x) \frac{\partial^{2} v}{\partial x_{i} \partial x_{j}}+\sum_{i=1}^{N} b_{i}(x) \frac{\partial v}{\partial x_{i}}\right)=\lambda(m(x)+g(x)) v & \text { in } B_{R}, \\
v=1 & \text { on } \partial B_{R}
\end{array}
$$

has a unique solution $\hat{v}(x ; \lambda) \in C^{2+\alpha}\left(\bar{B}_{R}\right)$ for every $\lambda<\lambda_{2}$ ([1, Theorem 4.4]). From the maximum principle it follows that for $0<\lambda<\lambda_{2}$

$$
\begin{array}{ll}
1 \equiv \hat{v}(x ; 0) \leqq \hat{v}(x ; \lambda), & x \in B_{R}, \\
\frac{\partial \hat{v}(x ; \lambda)}{\partial \nu}<0, & x \in \partial B_{R},
\end{array}
$$

where $\nu=\left(\nu_{1}, \cdots, \nu_{N}\right)$ is a conormal vector to $\partial B_{R}$ whose components are

$$
\nu_{i}=\left(\sum_{i=1}^{N}\left(\sum_{j=1}^{N} a_{i j}(x) x_{j} / R\right)^{2}\right)^{-1 / 2} \sum_{j=1}^{N} a_{i j}(x) x_{j} / R, \quad i=1, \cdots, N .
$$

Furthermore, by the $L^{p}$-estimates for the solution $\tilde{v}(x)=\hat{v}(x ; \lambda)-1$ of

$$
\begin{array}{rlrl}
-\left(\sum_{i, j=1}^{N} a_{i j}(x) \frac{\partial^{2} \tilde{v}}{\partial x_{i} \partial x_{j}}+\sum_{i=1}^{N} b_{i}(x) \frac{\partial \tilde{v}}{\partial x_{i}}\right) & =\lambda(m(x)+g(x)) \hat{v}(x ; \lambda) & & \text { in } B_{R} \\
\tilde{v} & =0 & \text { on } \partial B_{R}
\end{array}
$$

and Sobolev's imbedding theorem we obtain

$$
\chi(\lambda) \equiv \max _{|x|=R}\left|\frac{\partial \hat{v}(x ; \lambda)}{\partial \nu}\right|=O(\lambda) \quad \text { as } \lambda \rightarrow 0
$$

and so $\chi(\lambda) \leqq M \lambda, 0 \leqq \lambda<\lambda_{2}$, for some $M>0$ (see e.g. the proof of Theorem 4.3 in [9]).

Put now

$$
\tilde{\lambda}=\min \left\{\lambda_{1}, \lambda_{2}, 1 /(2 \kappa), \hat{a} /\left(4 M p_{*}(R) \pi_{*}(R)\right)\right\},
$$

where $\kappa=\int_{R}^{\infty} \pi_{*}(r) p_{*}(r) m^{*}(r) d r$ and $\hat{a}=\min _{|x|=R}\left\{a(x)\left(\sum_{i=1}^{N}\left(\sum_{j=1}^{N} a_{i j}(x) x_{j} / R\right)^{2}\right)^{-1 / 2}\right\}$. Then, we have

$$
\chi(\lambda) \leqq \frac{\hat{a}}{4 p_{*}(R) \pi_{*}(R)} \quad \text { for } 0 \leqq \lambda<\tilde{\lambda}
$$

Let $0<\lambda<\tilde{\lambda}$ and choose a constant $\xi$ so that

$$
\xi>\max \left\{4 \sigma, \frac{1}{\lambda}\right\}
$$

where $\sigma=\int_{R}^{\infty} \pi_{*}(r) p_{*}(r) g *(r) d r$. Since $\lambda<1 / 2 \kappa$ and $\xi>\sigma /(1-\lambda \kappa)$, by Lemma 3.3 
and Remark 3.1 , we can take an $\eta(\xi)>0$ such that the solution $y(r ; \eta(\xi))$ of the problem

$$
\begin{aligned}
& \left(p_{*}(r) y^{\prime}\right)^{\prime}+\lambda p_{*}(r) m *(r) y+p_{*}(r) g^{*}(r)=0, \quad r>R, \\
& y(R)=\xi, \quad y^{\prime}(R)=-\eta(\xi)
\end{aligned}
$$

satisfies $y(r ; \eta(\xi))>0, y^{\prime}(r ; \eta(\xi))<0$ for $r>R$ and $\lim _{r \rightarrow \infty} y(r ; \eta(\xi))=0$. From Remark 3.1 and (3.22) it follows that

$$
\eta(\xi) \geqq \frac{\xi(1-\tilde{\lambda} \kappa)-\sigma}{p_{*}(R) \pi_{*}(R)} \geqq \frac{\xi}{4 p_{*}(R) \pi_{*}(R)} .
$$

For $0<\lambda<\tilde{\lambda}$, define a function $v$ by

$$
v(x)=\xi \hat{v}(x ; \lambda) \quad \text { for } \quad x \in \bar{B}_{R} ; \quad v(x)=y(|x| ; \eta(\xi)) \text { for } x \in R^{N} \backslash \bar{B}_{R} .
$$

Then $v$ is a supersolution of (3.17). In fact, $v$ is continuous in $R^{N}$ and satisfies

$$
\begin{aligned}
& L v \geqq \lambda(m(x)+g(x)) v=\lambda m(x) v+\lambda \xi g(x) \hat{v}(x ; \lambda) \\
& \quad \geqq \lambda m(x) v+g(x) \quad \text { in } B_{R}
\end{aligned}
$$

by (3.19), (3.20) and (3.22). Furthermore we see that

$$
\begin{aligned}
L v & =-a(x) y^{\prime \prime}(r)-b(x) y^{\prime}(r)+c(x) y(r) \\
& =-a(x)\left(y^{\prime \prime}(r)+b_{*}(r) y^{\prime}(r)\right)+\left(a(x) b_{*}(r)-b(x)\right) y^{\prime}(r)+c(x) y(r) \\
& \geqq a(x)\left(\lambda m^{*}(r) y(r)+g^{*}(r)\right) \geqq \lambda m(x) v+g(x) \quad \text { in } R^{N} \backslash \bar{B}_{R},
\end{aligned}
$$

where $y(r)=y(r ; \eta(\xi)), r=|x|$, and we have used the relation $y^{\prime}(r)<0$ for $r>R$ and the reformulation of (3.23)

$$
y^{\prime \prime}+b_{*}(r) y^{\prime}+\lambda m^{*}(r) y+g^{*}(r)=0, \quad r>R .
$$

Finally, by (3.21) and (3.24), $v_{1}=\left.v\right|_{\bar{B}_{R}}$ and $v_{2}=\left.v\right|_{\boldsymbol{R}^{N} \backslash \boldsymbol{B}_{R}}$ satisfy

$$
\begin{aligned}
\frac{\partial v_{1}}{\partial \nu}-\frac{\partial v_{2}}{\partial \nu} & =\xi \frac{\partial v(x ; \lambda)}{\partial \nu}-\frac{\partial y(|x| ; \eta(\xi))}{\partial \nu} \\
& \geqq-\xi \chi(\lambda)-y^{\prime}(R ; \eta(\xi)) a(x)\left(\sum_{i=1}^{N}\left(\sum_{j=1}^{N} a_{i j}(x) x_{j} /|x|\right)^{2}\right)^{-1 / 2} \\
& \geqq-\xi \chi(\lambda)+\eta(\xi) \hat{a} \\
& \geqq-\frac{\xi \hat{a}}{4 p_{*}(R) \pi_{*}(R)}+\frac{\xi \hat{a}}{4 p_{*}(R) \pi_{*}(R)}=0 \quad \text { on } \partial B_{R} .
\end{aligned}
$$

Thus $v(x)$ is a positive supersolution of (3.17) tending to 0 as $|x| \rightarrow \infty$. Note that this $v$ is also a supersolution of (3.17) with $\lambda=0$.

Let us construct a subsolution of (3.17). By the hypothesis for $g$ we may 
assume that $g(x)>0$ in $\bar{B}$ for some ball $B$ in $R^{N}$. Let $\mu_{0}>0$ be the principal eigenvalue of the operator $L$ in $B$ with the Dirichlet boundary condition and let $\varphi_{0} \in C^{2+\alpha}(\bar{B})$ be an eigenfunction corresponding to $\mu_{0}$ so chosen that

$$
0<\varphi_{0}(x) \leqq \min \left\{g(x) / \mu_{0}, v(x)\right\}, \quad x \in B .
$$

Then,

$$
\begin{aligned}
& L \varphi_{0}(x)=\mu_{0} \varphi_{0}(x) \leqq g(x) \leqq \lambda m(x) \varphi_{0}(x)+g(x) \quad \text { in } B, \\
& \varphi_{0}(x)=0 \text { and } \frac{\partial \varphi_{0}(x)}{\partial \nu}<0 \text { on } \partial B,
\end{aligned}
$$

and so the function $w$ defined by

$$
w(x)=\varphi_{0}(x) \text { for } \quad x \in \bar{B} ; \quad w(x)=0 \text { for } x \in \boldsymbol{R}^{N} \backslash B
$$

is a subsolution of (3.17) satisfying $w(x) \leqq v(x)$ in $\boldsymbol{R}^{N}$. Hence, by Lemma 3.1 (3.17) has a solution $u$ such that $w(x) \leqq u(x) \leqq v(x)$ in $\boldsymbol{R}^{N}$ and $\lim _{|x| \rightarrow \infty} u(x)=0$. Since $L u \geqq 0, u(x) \geqq 0$ in $\boldsymbol{R}^{N}$ and $u(x)>0$ in $B$, this $u$ is positive throughout $\boldsymbol{R}^{N}$ by the maximum principle.

To prove the uniqueness, let $u_{1}$ and $u_{2}$ be two solutions of (3.17) for $0 \leqq \lambda$ $<\tilde{\lambda}$ which tend to 0 as $|x| \rightarrow \infty$. Then $\tilde{u}=u_{1}-u_{2}$ satisfies

$$
L \tilde{u}=\lambda m(x) \tilde{u} \text { in } \boldsymbol{R}^{N}, \quad \lim _{|x| \rightarrow \infty} \frac{\tilde{u}(x)}{V(x)}=\lim _{|x| \rightarrow \infty} \tilde{u}(x)=0,
$$

where $V$ is a function satisfying (3.18). Applying Lemma 3.2, we see that $\tilde{u}(x)=u_{1}(x)-u_{2}(x) \geqq 0$ in $\boldsymbol{R}^{N}$. The same procedure yields $u_{2}(x)-u_{1}(x) \geqq 0$ in $\boldsymbol{R}^{N}$, and consequently we have $u_{1}(x)=u_{2}(x)$ in $\boldsymbol{R}^{N}$.

Corollary 3.1. Let $N \geqq 3$ and let $c, m$ and $g$ be nonnegative locally Hölder continuous functions in $\boldsymbol{R}^{N}$ with exponent $\alpha, 0<\alpha<1$. Suppose that $m(x)>0, g(x) \equiv 0$ in $R^{N}$, and

$$
\int_{R}^{\infty} r\{m *(r)+g *(r)\} d r<\infty
$$

for some $R>0$, where $m *(r)=\max _{|x|=r} m(x)$ and $g^{*}(r)=\max _{|x|=r} g(x)$.

Then, there is a $\tilde{\lambda}>0$ such that, for every $\lambda \in[0, \tilde{\lambda})$, the equation

$$
-\Delta u+c(x) u=\lambda m(x) u+g(x) \quad \text { in } R^{N}
$$

possesses a unique entire positive solution $u \in C_{l o c}^{2+\alpha}\left(\boldsymbol{R}^{N}\right)$ satisfying $\lim _{|x| \rightarrow \infty} u(x)$ $=0$. Moreover, if

$$
\int_{R}^{\infty} r^{1+\varepsilon}\{m *(r)+g *(r)\} d r<\infty
$$


for some $\varepsilon \in(0, N-2]$, then the above solution $u$ satisfies $u(x)=O\left(|x|^{-\varepsilon}\right)$ as $|x|$ $\rightarrow \infty$.

Proof. Since the existence of $\tilde{\lambda}>0$ with the desired property is guaranteed by Lemma 3.4, we need only to prove the last statement. From the proof of Lemma 3.4 it suffices to show that under (3.27) a positive solution $y$ of the ordinary differential equation

$$
\left(r^{N-1} y^{\prime}\right)^{\prime}+r^{N-1}\left(\lambda m^{*}(r) y+g^{*}(r)\right)=0, \quad r>R,
$$

satisfies $y(r)=O\left(r^{-\varepsilon}\right)$ as $r \rightarrow \infty$, provided $\lim _{r \rightarrow \infty} y(r)=0$. Integrating (3.28) twice and using the boundedness of $y$, we have

$$
\begin{aligned}
& y(r)=-(N-2)^{-1} R^{N-1} y^{\prime}(R) r^{-(N-2)}+\lambda \int_{r}^{\infty} s^{-(N-1)}\left(\int_{R}^{s} t^{N-1} m^{*}(t) y(t) d t\right) d s \\
&+\int_{r}^{\infty} s^{-(N-1)}\left(\int_{R}^{s} t^{N-1} g^{*}(t) d t\right) d s \\
& \leqq-(N-2)^{-1} R^{N-1} y^{\prime}(R) r^{-(N-2)}+M \int_{r}^{\infty} s^{-(N-1)}\left(\int_{R}^{s} t^{N-1}\left(m^{*}(t)+g^{*}(t)\right) d t\right) d s, \\
& \quad r>R
\end{aligned}
$$

for some constant $M>0$. It is not hard to show that

$$
J(r) \equiv \int_{r}^{\infty} s^{-(N-1)}\left(\int_{R}^{s} t^{N-1}\left(m^{*}(t)+g^{*}(t)\right) d t\right) d s=O\left(r^{-\varepsilon}\right) \quad \text { as } r \rightarrow \infty
$$

by using the identity

$$
J(r)=\int_{r}^{\infty} s^{-(N-1)}\left(\int_{R}^{s} t^{N-2-\varepsilon}\left(\int_{R}^{t} \tau^{1+\varepsilon}\left(m^{*}(\tau)+g^{*}(\tau)\right) d \tau\right)^{\prime} d t\right) d s, \quad r>R,
$$

L'Hospital's rule and (3.27). (3.29) and (3.30) imply $y(r)=O\left(r^{-\varepsilon}\right)$ as $r \rightarrow \infty$.

\section{§4. Proofs of Theorems 2.1 and 2.2}

Proof of Theorem 2.1. Let $v \in C_{l o c}^{2+\alpha}\left(\boldsymbol{R}^{N}\right)$ be a positive solution of the linear equation $L v=m(x)$ in $R^{N}$ such that $\lim _{|x| \rightarrow \infty} v(x)=0$. Such a $v$ exists by Lemma 3.4. Put $\lambda^{*}=1 / C_{U}$ and let $0<\lambda<\lambda^{*}$, where $C_{U}>0$ is the constant corresponding to $U=\sup _{x \in R^{N}} v(x)$ in $\left(F_{2}\right)$. Then, $v$ is obviously a supersolution of (1.1) by $\left(F_{2}\right)$.

To construct a subsolution, choose a ball $B \subset \bar{B} \subset \Omega_{0}$, let $\mu_{0}>0$ be the principal eigenvalue of $L$ on $B$ with the Dirichlet boundary condition, and $\varphi_{0}$ be a positive eigenfunction corresponding to $\mu_{0}$ satisfying

$$
0<\varphi_{0}(x) \leqq \min \left\{u_{0}, v(x)\right\}, \quad x \in B,
$$


where $u_{0}$ is a positive constant so chosen that

$$
\frac{f(x, u)}{u} \geqq \frac{\mu_{0}}{\lambda} \quad \text { for } x \in \bar{B}, 0<u \leqq u_{0} .
$$

Then, it is easy to verify that the function $w$ defined by

$$
w(x)=\varphi_{0}(x) \text { for } x \in \bar{B} ; \quad w(x)=0 \text { for } x \in R^{N} \backslash B
$$

is a subsolution of (1.1) with the property $w(x) \leqq v(x)$ in $\boldsymbol{R}^{N}$. Hence, by Lemma 3.1 (1.1) has a solution $u$ satisfying $w(x) \leqq u(x) \leqq v(x)$ in $\boldsymbol{R}^{N}$. Because of $\left(F_{2}\right)$ the positivity of $u$ follows from the maximum principle as in the proof of Lemma 3.4. This completes the proof.

REMARK 4.1. It may happen that $\lambda^{*}$ in Theorem 2.1 is finite. This is the case if, for example,

$$
c_{0} \equiv \inf _{x \in \Omega, u>0} \frac{f(x, u)}{u}>0
$$

for some bounded domain $\Omega$ in $R^{N}$. In this case (1.1) has no positive solution provided $\lambda(>0)$ is sufficiently large. In fact, assume that (1.1) has a positive solution $u$ for $\lambda>0$. Then $u$ satisfies

$$
L u=\lambda f(x, u) \geqq \lambda c_{0} u \quad \text { in } B^{\prime}, \quad u>0 \quad \text { on } \partial B^{\prime}
$$

for some ball $B^{\prime} \subset \bar{B}^{\prime} \subset \Omega$. Hence, $\lambda c_{0}$ cannot be greater than the principal eigenvalue $\tilde{\mu}$ of $L$ on $B^{\prime}$ with Dirichlet boundary condition (see e.g. [1, p. 636]), i.e., $\lambda \leqq \tilde{\mu} / c_{0}$. This implies that (1.1) has no positive solution for $\lambda>\tilde{\mu} / c_{0}$.

Proof of Theorem 2.2. The proof of (i) is essentially similar to those of Theorem 2.1 and of (ii) below, and will be omitted.

(ii) Let $\tilde{\lambda}>0$ be a constant such that, for every $\lambda \in[0, \tilde{\lambda})$, there exists a unique entire positive solution of

$$
L u=\lambda m(x) u+g(x) \quad \text { in } R^{N}, \lim _{|x| \rightarrow \infty} u(x)=0,
$$

and let $\lambda_{1}>0$ be a constant for which there is a function $V \in C^{2}\left(R^{N}\right)$ satisfying

$$
L V(x) \geqq \lambda_{1} m(x) V(x), \quad 0<M_{1} \leqq V(x) \leqq M_{2}<\infty \quad \text { in } R^{N},
$$

for some constants $M_{1}$ and $M_{2}$, where $m$ and $g$ are in $\left(F_{4}\right)$. The existence of $\tilde{\lambda}$ and $\lambda_{1}$ is guaranteed by (2.3), Lemma 3.4 and Theorem 4.3 of [9].

Denote $\lambda_{0}=\lambda_{0}(\tilde{m})$ and let $\lambda>\lambda_{0}$. Choosing $\mu>0$ so small that $\lambda \mu<$ $\min \left\{\tilde{\lambda}, \lambda_{1}\right\}$, we can obtain a positive function $\hat{v} \in C_{l o c}^{2+\alpha}\left(\boldsymbol{R}^{N}\right)$ such that

$$
L \hat{v}=\lambda \mu m(x) \hat{v}+g(x) \quad \text { in } \boldsymbol{R}^{N}, \lim _{|x| \rightarrow \infty} \hat{v}(x)=0 .
$$


The function $v(x)=\lambda C(\mu) \hat{v}(x)$ satisfies

$$
L v=\lambda(\mu m(x) v+C(\mu) g(x)) \quad \text { in } R^{N}, \lim _{|x| \rightarrow \infty} v(x)=0,
$$

where $C(\mu)$ is a positive constant in $\left(F_{4}\right)$. By (4.3) and $\left(F_{4}\right)$ this $v$ is a supersolution of (1.1). Next, by the definition of $\lambda_{0}$, we can choose $n \geqq 1$ such that the principal eigenvalue $\lambda_{n}(\tilde{m})$ of $(2.2)$ satisfies $\lambda_{0} \leqq \lambda_{n}(\tilde{m})<\lambda$. From $\left(F_{5}\right)$ it follows that

$$
\lambda f(x, u) \geqq \lambda_{n}(\tilde{m}) \tilde{m}(x) u \quad \text { for } x \in R^{N}, 0<u \leqq u_{0}
$$

for sufficiently small $u_{0}>0$. Take now a positive eigenfunction $\varphi_{n}$ of (2.2) corresponding to $\lambda_{n}(\tilde{m})$ which satisfies

$$
0<\varphi_{n}(x) \leqq \min \left\{u_{0}, v(x)\right\}, \quad x \in B_{n} .
$$

By this and (4.4) the function $w$ defined by

$$
w(x)=\varphi_{n}(x) \text { for } x \in \bar{B}_{n} ; w(x)=0 \text { for } x \in \boldsymbol{R}^{N} \backslash B_{n}
$$

is a subsolution of (1.1) satisfying $w(x) \leqq v(x)$ in $\boldsymbol{R}^{N}$. So by Lemma 3.1 there is a solution $u$ of (1.1) such that

$$
0<u(x) \leqq v(x) \text { in } R^{N} \text { and } \lim _{|x| \rightarrow \infty} u(x)=0 .
$$

Therefore, (1.1) has an entire positive solution tending to 0 as $|x| \rightarrow \infty$ for $\lambda>\lambda_{0}$. Since $\lambda>\lambda_{0}$ is arbitrary, we conclude that $\lambda^{*}=\infty$ and $\lambda_{*} \leqq \lambda_{0}$.

To prove the positivity of $\lambda_{*}$ under condition (2.4), put

$$
\tilde{c}=\sup _{x \in R^{N}, u>0} f(x, u) / m(x) u
$$

and assume that, for some $\lambda \in\left(0, \lambda_{1} / \tilde{c}\right),(1.1)$ has a positive solution $u$ satisfying $\lim _{|x| \rightarrow \infty} u(x)=0$. Then, noting that this $u$ satisfies

$$
\begin{aligned}
& L u=\lambda f(x, u) \leqq \lambda \tilde{c} m(x) u \leqq \lambda_{1} m(x) u \quad \text { in } R^{N}, \\
& \lim _{|x| \rightarrow \infty} \frac{u(x)}{V(x)}=0,
\end{aligned}
$$

where $V$ is as in (4.2), we get $u(x) \leqq 0$ in $R^{N}$ by Lemma 3.2, a contradiction. This shows that $\lambda_{*} \geqq \lambda_{1} / \tilde{c}>0$.

Finally, we claim the existence of a maximal decaying positive solution of (1.1) for every $\lambda>\lambda_{*}$. To this end, let $\lambda>\lambda_{*}$ and take a constant $\mu^{*}>0$ so that $\lambda \mu^{*}<\min \left\{\tilde{\lambda}, \lambda_{1}\right\}$. Let $\bar{v}$ be the unique positive solution of

$$
L \bar{v}=\lambda \mu^{*} m(x) \bar{v}+\lambda C\left(\mu^{*}\right) g(x) \quad \text { in } R^{N}
$$

satisfying $\lim _{|x| \rightarrow \infty} \bar{v}(x)=0$, where $C\left(\mu^{*}\right)$ is as in $\left(F_{4}\right)$. Then, repeating the 
same procedure as above, by Lemma 3.1 we obtain a positive solution $\bar{u}$ of (1.1) such that

$$
u(x) \leqq \bar{u}(x) \leqq \bar{v}(x) \quad \text { in } R^{N}
$$

for any solution $u$ satisfying $0 \leqq u(x) \leqq \bar{v}(x)$ in $R^{N}$. This solution $\bar{u}$ is the desired maximal decaying entire positive solution of (1.1). To show this, let $u$ be an arbitrary positive solution of (1.1) tending to 0 as $|x| \rightarrow \infty$. Then, by (1.1) and $\left(F_{4}\right)$,

$$
L u=\lambda f(x, u(x)) \leqq \lambda \mu^{*} m(x) u(x)+\lambda C\left(\mu^{*}\right) g(x) \quad \text { in } \boldsymbol{R}^{v},
$$

and so $\bar{w}(x)=\bar{v}(x)-u(x)$ satisfies

$$
L \bar{w} \geqq \lambda \mu^{*} m(x) \bar{w} \text { in } R^{N} \text { and } \lim _{|x| \rightarrow \infty} \frac{\bar{w}(x)}{V(x)}=0
$$

for the above mentioned function $V$. Lemma 3.2 then implies that $\bar{w}(x) \geqq 0$, i.e., $u(x) \leqq \bar{v}(x)$ in $\boldsymbol{R}^{N}$. From this and (4.5) it follows that $u(x) \leqq \bar{u}(x)$ in $\boldsymbol{R}^{N}$. Thus the proof is complete.

Corollary 4.1. Assume that $(H)$ holds, and let $\Phi$ be a locally Hölder continuous positive function in $\boldsymbol{R}^{N}$. If $0 \leqq \gamma<1$ and

$$
\int_{R}^{\infty} \pi_{*}(r) p_{*}(r) \Phi^{*}(r) d r<\infty
$$

for some $R>0$, then

$$
L u=\Phi(x) u^{r} \quad \text { in } R^{N}
$$

possesses a maximal decaying entire positive solution $\vec{u}$.

Proof. Using the inequality $u^{r} \leqq \mu u+(1-\gamma)(\gamma / \mu)^{r /(1-\gamma)}$ for $\mu>0, u \geqq 0$ and $0<\gamma<1$, we easily see that the function $f(x, u)=\Phi(x) u^{r}$ satisfies all the conditions in Theorem 2.2. Therefore, the assertion follows from (i) of Theorem 2.2 .

This corollary is a partial extension of [7, Theorem 1] dealing with the case $L=-\Delta$ and $c(x) \equiv 0$ in $R^{N}, N \geqq 3$.

As a byproduct of Theorem 2.2 we obtain the following uniqueness result.

Corollary 4.2. Assume that $(H),\left(F_{1}\right),\left(F_{3}\right),\left(F_{4}\right)$ and $(2.3)$ are satisfied and that $f(x, u)$ is nonincreasing in $u>0$ for any fixed $x \in R^{N}$. Then, for every $\lambda>0,(1.1)$ has a unique entire positive solution $u$ satisfying $\lim _{|x| \rightarrow \infty} u(x)=0$.

Proof. By (i) of Theorem 2.2 (1.1) has a maximal decaying entire 
positive solution $\bar{u}$ for every $\lambda>0$. To prove the uniqueness let $u$ be any entire positive solution of (1.1) such that $\lim _{|x| \rightarrow \infty} u(x)=0$. Then, since $f(x, u)$ is nonincreasing in $u>0$ and $\bar{u}(x) \geqq u(x)$ in $R^{N}, \tilde{u}(x)=\bar{u}(x)-u(x)$ satisfies

$$
L \tilde{u}=\lambda(f(x, \bar{u})-f(x, u)) \leqq 0 \text { in } R^{N} \text { and } \lim _{|x| \rightarrow \infty} \tilde{u}(x)=0 .
$$

This and Lemma 3.2 show that $\tilde{u}(x) \leqq 0$ in $\boldsymbol{R}^{N}$. Hence we see that $\tilde{u}(x)=0$, i.e., $u(x)=\bar{u}(x)$ throughout $\boldsymbol{R}^{N}$, proving the uniqueness.

REMARK 4.2. Let $B_{*}, C^{*}, M^{*}$ and $G^{*}$ be locally Hölder continuous functions in $\boldsymbol{R}_{+}$satisfying

$$
B_{*}(r) \leqq b_{*}(r), \quad c^{*}(r) \leqq C^{*}(r), \quad m *(r) \leqq M^{*}(r) \quad \text { and } \quad g^{*}(r) \leqq G^{*}(r), \quad r>0,
$$

respectively, and put $P_{*}(r)=\exp \left(\int_{R}^{r} B_{*}(s) d s\right), r>0$. Suppose that $\int_{R}^{\infty} d s / P_{*}(s)$ $<\infty$ instead of condition $(H)$ and put $\Pi_{*}(r)=\int_{r}^{\infty} d s / P_{*}(s), r>0$. Then, it is easy to see that all the conclusions of Theorems 2.1 and 2.2 remain true if in the hypotheses of these theorems $p_{*}, \pi_{*}, c^{*}, m^{*}$ and $g^{*}$ are replaced by $P_{*}$, $\Pi_{*}, C^{*}, M^{*}$ and $G^{*}$, respectively.

EXAMPLE 4.1. Suppose that the coefficients $a_{i j}$ and $b_{i}$ of $L$ are bounded in $\boldsymbol{R}^{N}$ and satisfy

$$
\liminf _{|x| \rightarrow \infty} \sum_{i=1}^{N} b_{i}(x) x_{i} /|x|>0 .
$$

If $f(x, u)$ satisfies $\left(F_{1}\right)-\left(F_{5}\right)$, and if

$$
\int_{R}^{\infty}\left\{m^{*}(r)+g^{*}(r)\right\} d r<\infty
$$

for some $R>0$, where $m *(r)=\max _{|x|=r} m(x)$ and $g *(r)=\max _{|x|=r} g(x)$, then the conclusions in Theorems 2.1 and 2.2 still hold. In fact, by (4.6) and the boundedness of the coefficient of $L$ we can choose a constant $\beta_{*}>0$ so that $B_{*}(r) \equiv \beta_{*}$ satisfies $B_{*}(r) \leqq b_{*}(r)$ for $r>R$ provided $R>0$ is sufficiently large. Since

$$
P_{*}(r)=\exp \left(\int_{R}^{r} B_{*}(s) d s\right)=e^{f_{*}(r-R)}, \quad r>R
$$

satisfies

$$
\int_{R}^{\infty} \frac{d r}{P_{*}(r)}<\infty, \int_{R}^{\infty}\left(\int_{r}^{\infty} \frac{d s}{P_{*}(s)}\right) P_{*}(r)\left\{m^{*}(r)+g^{*}(r)\right\} d r<\infty
$$


by the positivity of $\beta_{*}$ and (4.7), the assertion follows from Remark 4.2.

\section{§. The case $L=-\Delta+c$}

In this section, we treat (1.1) in the case where $L=-\Delta+c$, i.e.,

$$
-\Delta u+c(x) u=\lambda f(x, u) \quad \text { in } R^{N}, N \geqq 3 .
$$

We first consider the uniqueness of decaying entire positive solution of (5.1). For that purpose the following conditions $\left(F_{5}^{\prime}\right)$ and $\left(F_{6}\right)$ are imposed on $f$.

$\left(F_{5}^{\prime}\right)$ There are a positive function $\tilde{m} \in C_{l o c}^{\alpha}\left(\boldsymbol{R}^{N}\right), 0<\alpha<1$, and a constant $\gamma, 0 \leqq \gamma \leqq 1$, such that

$$
\lim _{u \rightarrow 0+} \frac{f(x, u)}{\tilde{m}(x) u^{\gamma}}=1 \quad \text { uniformly in } R^{N} .
$$

$\left(F_{b}\right) \quad f(x, u) / u$ is strictly decreasing in $u>0$ for any fixed $x \in R^{N}$.

Throughout this section we employ the notation $h^{*}(r)=\max _{|x|=r} h(x)$, $r>0$, for a continuous function $h$ in $\boldsymbol{R}^{N}$ (cf. Section 2).

Theorem 5.1. Assume that $N \geqq 3$ and $c$ is nonnegative and locally Hölder continuous with exponent $\alpha, 0<\alpha<1$, in $R^{N}$, and $f$ satisfies $\left(F_{1}\right),\left(F_{4}\right),\left(F_{5}^{\prime}\right)$ and $\left(F_{6}\right)$. If

$$
\int_{R}^{\infty} r^{(N+r-1) /(r+1)}\left\{c^{*}(r)+m^{*}(r)+g *(r)\right\} d r<\infty
$$

for some $R>0$, then there is a $\lambda_{*} \geqq 0$ such that, for every $\lambda>\lambda_{*}$, (5.1) has a unique positive solution $u$ satisfying $\lim _{|x| \rightarrow \infty} u(x)=0$.

Proof. In view of $\left(F_{1}\right),\left(F_{4}\right)$ and $\left(F_{5}^{\prime}\right)$ Theorem 2.2 guarantees the existence of a $\lambda_{*}>0$ such that, for every $\lambda>\lambda_{*},(5.1)$ has a miximal decaying entire positive solution $\bar{u}$. We prove the uniqueness by using the idea of [2], [4] and [8]. Let $u$ be any positive solution of (5.1) such that $\lim _{|x| \rightarrow \infty} u(x)=0$. Then, from the proofs of Theorem 2.2 and Corollary 3.1 we see that $u$ and $\bar{u}$ satisfy

$$
u(x), \bar{u}(x)=O\left(|x|^{-\varepsilon}\right) \quad \text { as }|x| \rightarrow \infty,
$$

where $\varepsilon=(N-2) /(\gamma+1)$. Take a function $\psi \in C^{\infty}\left(\overline{\boldsymbol{R}}_{+}\right)$such that

$$
\psi(r)=1 \text { for } 0 \leqq r \leqq 1 ; 0<\psi(r)<1 \text { for } 1<r<2 ; \psi(r)=0 \text { for } r \geqq 2
$$

and put $\psi_{\rho}(r)=\psi(r / \rho)$ for $\rho>0$. Then, by (5.1), $\left(F_{6}\right)$ and the relation $0<u(x)$ $\leqq \bar{u}(x), x \in R^{N}$, we get 


$$
\begin{aligned}
(\bar{u}(x) \Delta u(x)-u(x) \Delta \bar{u}(x)) \psi_{\rho}(|x|) & =\lambda\{f(x, \bar{u}) / \bar{u}-f(x, u) / u\} u(x) \bar{u}(x) \psi_{\rho}(|x|) \\
& \leqq 0 \quad \text { in } R^{N} .
\end{aligned}
$$

Integrating the above over $\boldsymbol{R}^{N}$ and applying Green's theorem, we obtain

$$
\begin{aligned}
I_{\rho} & \equiv \int_{R^{N}}(u(x) \nabla \bar{u}(x)-\bar{u}(x) \nabla u(x)) \cdot \nabla \psi_{\rho}(|x|) d x \\
& =\lambda \int_{R^{N}}(f(x, \bar{u}) / \bar{u}-f(x, u) / u) u(x) \bar{u}(x) \psi_{\rho}(|x|) d x \quad \rho>0,
\end{aligned}
$$

where $\nabla u(x)$ denotes the gradient of $u(x)$. The choice of $\psi_{\rho}$ shows that

$$
I_{\rho}=\int_{\rho \leqq|x| \leqq 2 \rho}(u(x) \nabla \bar{u}(x)-\bar{u}(x) \nabla u(x)) \cdot \nabla \psi_{\rho}(|x|) d x .
$$

It can be shown that $u$ and $\bar{u}$ satisfy
(a) $u, \bar{u} \in L_{l o c}^{1}\left(\boldsymbol{R}^{N}\right)$,
(b) $\Delta u, \Delta \bar{u} \in L^{1}\left(\boldsymbol{R}^{N}\right)$, and
(c) $\lim _{n \rightarrow \infty} \int_{1 \leqq|x| \leqq 2} u(n x) d x=\lim _{n \rightarrow \infty} \int_{1 \leqq|x| \leqq 2} \bar{u}(n x) d x=0$.

We sketch only the proof of (b) for $u$. Since $\lim _{|x| \rightarrow \infty} u(x)=0$, by $\left(F_{5}^{\prime}\right)$ there is $R_{1}>0$ such that $0 \leqq f(x, u(x)) \leqq 2 \tilde{m}(x) u(x)^{\gamma}$ for $|x|>R_{1}$. Using this and (5.4), we have

$$
|\Delta u(x)|=c(x) u+\lambda f(x, u(x)) \leqq M_{1}\left\{c(x)|x|^{-\varepsilon}+\tilde{m}(x)|x|^{-\varepsilon r}\right\}, \quad|x|>R_{1}
$$

for some constant $M_{1}>0$. Since by $\left(F_{4}\right),(5.2)$ and $(5.3) \tilde{m}^{*}(r)$ satisfies $\int_{R_{1}}^{\infty} r^{1+\varepsilon} \tilde{m}^{*}(r) d r<\infty$, from (5.6) and (5.3) it follows that

$$
\begin{aligned}
\int_{R^{N}}|\Delta u(x)| d x & =\left(\int_{|x| \leqq R_{\mathbf{1}}}+\int_{|x|>R_{\mathbf{1}}}\right)|\Delta u(x)| d x \\
& \leqq \int_{|x| \leqq R_{1}}|\Delta u(x)| d x+M_{1} \int_{|x|>R_{\mathbf{1}}}\left\{c(x)|x|^{-\varepsilon}+\tilde{m}(x)|x|^{-\varepsilon r}\right\} d x \\
& \leqq \int_{|x| \leqq R_{1}}|\Delta u(x)| d x+M_{2} \int_{R_{1}}^{\infty}\left\{r^{N-1-\varepsilon} c^{*}(r)+r^{N-1-\varepsilon r} \tilde{m}^{*}(r)\right\} d r<\infty,
\end{aligned}
$$

where $M_{2}>0$ is a constant.

From (a)-(c) and [2, Lemma A.5] it follows that

$$
\int_{\rho \leqq|x| \leqq 2 \rho}(|\nabla u(x)|+|\nabla \bar{u}(x)|) d x=O(\rho) \quad \text { as } \rho \rightarrow \infty,
$$

which, combined with (5.4) and the fact that $\left|\nabla \psi_{\rho}(|x|)\right|=O\left(\rho^{-1}\right)$ as $\rho \rightarrow \infty$, implies that $\lim _{\rho \rightarrow \infty} I_{\rho}=0$. Therefore, letting $\rho \rightarrow \infty$ in (5.5), we obtain

$$
\int_{R^{N}}(f(x, \bar{u}) / \bar{u}-f(x, u) / u) u(x) \bar{u}(x) d x=0 .
$$


The nonpositivity of the integrand leads us to the equality $f(x, \bar{u}(x)) / \bar{u}(x)=$ $f(x, u(x)) / u(x)$ in $R^{N}$. Hence, by $\left(F_{6}\right)$ we conclude that $u(x)=\bar{u}(x)$ throughout $\boldsymbol{R}^{N}$. This finishes the proof.

Now we apply our theorems to the following two special equations:

$$
-\Delta u+c(x) u=\lambda\left(\Phi(x) u^{\gamma}+\Psi(x) u^{j}\right) \quad \text { in } \boldsymbol{R}^{N}, N \geqq 3
$$

and

$$
-\Delta u+c(x) u=\lambda\left(\Phi(x) u^{r}-\Psi(x) u^{\delta}\right) \quad \text { in } \boldsymbol{R}^{N}, N \geqq 3,
$$

where $\gamma$ and $\delta$ are nonnegative constants, $c, \Phi$ and $\Psi$ are nonnegative locally Hölder continuous functions in $\boldsymbol{R}^{N}$ with exponent $\alpha, 0<\alpha<1$, and especially $\Phi$ is positive throughout $\boldsymbol{R}^{N}$.

TheOREM 5.2. Let $0 \leqq \gamma<1, \gamma \leqq \delta$ and suppose that

$$
\int_{R}^{\infty} r\left\{\Phi^{*}(r)+\Psi^{*}(r)\right\} d r<\infty
$$

for some $R>0$. Then, there is a $\lambda^{*}>0$ such that, for every positive $\lambda<\lambda^{*},(5.7)$ has an entire positive solution $u$ satisfying $\lim _{|x| \rightarrow \infty} u(x)=0$. Furthermore, if $\Psi(x)>0$ in $\boldsymbol{R}^{N}$ and $\delta>1$, then the above $\lambda^{*}$ is finite and (5.7) has no decaying entire positive solution provided $\lambda>\lambda^{*}$.

Proof. The function $f(x, u)=\Phi(x) u^{\gamma}+\Psi(x) u^{\delta}$ satisfies $\left(F_{1}\right)-\left(F_{3}\right)$ with $m(x)$ $=\Phi(x)+\Psi(x)$ (see Example 2.1). Moreover, condition (5.9) implies (2.1). Therefore the assertion is a consequence of Theorem 2.1 and Remark 4.1.

TheOREM 5.3. Let $0 \leqq \gamma \leqq 1 \leqq \delta, \quad \gamma \neq \delta$, and suppose that $\Psi(x) / \Phi(x)$ is bounded in $\boldsymbol{R}^{N}$ and

$$
\int_{R}^{\infty} r \Phi^{*}(r) d r<\infty
$$

for some $R>0$.

(i) Let $0 \leqq \gamma<1$. For every $\lambda>0$, (5.8) has a maximal decaying entire positive solution.

(ii) Let $\gamma=1$. If $\inf _{x \in R^{N}} \Psi(x) / \Phi(x)>0$, then there is $a \lambda_{*}>0$ such that (5.8) has a maximal decaying entire positive solution for every $\lambda>\lambda_{*}$ but has no decaying entire positive solution for any positive $\lambda<\lambda_{*}$.

Furthermore, if $c$ and $\Phi$ satisfy

$$
\int_{R}^{\infty} r^{1+\varepsilon}\left\{c^{*}(r)+\Phi^{*}(r)\right\} d r<\infty, \quad \varepsilon=(N-2) /(\gamma+1),
$$

then (5.8) has at most one decaying entire positive solution for any $\lambda>0$. 
Proof. The function $f(x, u)=\Phi(x) u^{\gamma}-\Psi(x) u^{\delta}$ satisfies conditions $\left(F_{1}\right)$, $\left(F_{4}\right),\left(F_{5}^{\prime}\right)$ and $\left(F_{6}\right)$ with $m(x)=\tilde{m}(x)=g(x)=\Phi(x)$. The conditions (2.3) and (5.3) reduce to (5.10) and (5.11), respectively. Consequently, the assertion follows from Theorems 2.2 and 5.1.

REMARK 5.1. In the case where $0<\gamma<1$ and $c(x) \equiv \Psi(x) \equiv 0, x \in R^{N}$, that is, for the sublinear equation

$$
-\Delta u=\Phi(x) u^{r} . \quad \text { in } \boldsymbol{R}^{N}, N \geqq 3,0<\gamma<1,
$$

Fukagai [7, Theorems 1 and 2] proved the existence and uniqueness of decaying entire positive solutions under the condition (5.11) with $\varepsilon$ replaced by $\varepsilon=(1-\gamma)(N-2)$.

We conclude with some examples illustrating our theorems.

EXAMPLE 5.1. Let $N \geqq 3$. Let $c$ and $m$ satisfy that $c, m \in C_{l o c}^{\alpha}\left(\boldsymbol{R}^{N}\right), 0<\alpha$ $<1, c(x) \geqq 0$ and $m(x)>0$ in $\boldsymbol{R}^{N}$. Moreover, suppose that $\int_{R}^{\infty} r m^{*}(r) d r<\infty$ for some $R>0$.

(i) By (ii) of Theorem 2.2 the equation

$$
-\Delta u+c(x) u=\lambda m(x) \sin u \quad \text { in } \boldsymbol{R}^{N}
$$

has a maximal decaying entire positive solution for sufficiently large $\lambda>0$ but has no decaying entire positive solution for sufficiently small $\lambda>0$.

(ii) From (ii) of Theorem 2.2 and Theorem 5.1 it follows that if

$$
\int_{R}^{\infty} r^{N / 2}\left\{c^{*}(r)+m^{*}(r)\right\} d r<\infty,
$$

the equation

$$
-\Delta u+c(x) u=\lambda m(x) \log (1+u) \quad \text { in } \boldsymbol{R}^{N}
$$

has exactly one decaying entire positive solution for sufficiently large $\lambda>0$ but has no decaying entire positive solution provided $\lambda>0$ is sufficiently small.

(iii) Consider the equation

$$
-\Delta u+c(x) u=\lambda m(x) e^{K u} \quad \text { in } \boldsymbol{R}^{N},
$$

where $K$ is a nonzero constant.

(a) Let $K>0$. Theorem 2.1 implies that, for sufficiently small $\lambda>0$, (5.12) has a decaying entire positive solution.

(b) Let $K<0$. For every $\lambda>0$, (5.12) has exactly one decaying entire positive solution. This follows from (i) of Theorem 2.2 and Corollary 4.2. 


\title{
References
}

[1] H. Amann, Fixed point equations and nonlinear eigenvalue problems in ordered Banach spaces, SIAM Rev., 18 (1976), 346-384.

[2] P. Benilan, H. Brezis and M. G. Crandall, A semilinear equations in $L^{1}\left(\boldsymbol{R}^{N}\right)$, Ann. Scuola Norm. Sup. Pisa, 2 (1975), 523-555.

[ 3 ] H. Berestycki and P. L. Lions, Nonlinear scalar field equations, I, II, Arch. Rational Mech. Anal., 82 (1983), 313-345, 347-375.

[4] D. G. de Figueiredo, Positive solutions of semilinear elliptic problems, Lecture Notes in Math., 957 (1982), 34-87, Springer-Verlag.

[5] A. Friedman, Bounded entire solutions of elliptic equations, Pacific J. Math., 44 (1973), 497-507.

[6] N. Fukagai, On decaying entire solutions of second order sublinear elliptic equations, Hiroshima Math. J., 14 (1984), 551-562.

[ 7 ] N. Fukagai, Existence and uniqueness of entire solutions of second order sublinear elliptic equations, Funkcial. Ekvac., 29 (1986), 151-165.

[ 8 ] Y. Furusho and Y. Ogura, On the existence of bounded positive solutions of semilinear elliptic equations in exterior domains, Duke Math. J., 48 (1981), 497-521.

[9] Y. Furusho, Positive solutions of linear and quasilinear elliptic equations in unbounded domains, Hiroshima Math. J., 15 (1985), 173-220.

[10] E. Hille, Lectures on Ordinary Differential Equations, Addison-Wesley, 1969.

[11] N. Kawano, On bounded entire solutions of semilinear elliptic equations, Hiroshima Math. J., 14 (1984), 125-158.

[12] N. Kawano, J. Satsuma and S. Yotsutani, On the positive solutions of an Emdentype elliptic equation, Proc. Japan Acad., 61, Ser. A (1985), 186-189.

[13] T. Kusano and S. Oharu, Bounded entire solutions of second order semilinear elliptic equations with applications to a parabolic initial value problem, Indiana Univ. Math. J., 34 (1985), 85-95.

[14] T. Kusano and C. A. Swanson, Decaying entire positive solutions of quasilinear elliptic equations, Monatsh. Math., 101 (1986), 39-51.

[15] T. Kusano and W. F. Trench, Global existence of solutions of mixed sublinearsuperlinear differential equations, Hiroshima Math. J., 16 (1986), 597-606.

[16] W. M. Ni, On the elliptic equation $\Delta u+K(x) u^{(n+2) /(n-2)}=0$, its generalizations, and applications in geometry, Indiana Univ. Math. J., 31 (1982), 493-529.

[17] E. S. Noussair and C. A. Swanson, Global positive solutions of semilinear elliptic problems, Pacific J. Math., 115 (1984), 177-192.

[18] E. S. Noussair and C. A. Swanson, Decaying entire solutions of quasilinear elliptic equations, Funkcial. Ekvac., to appear.

[19] E. Z. Noussair and C. A. Swanson, Positive solutions of elliptic systems with bounded nonlinearities, to appear.

[20] M. H. Protter and H. F. Weinberger, Maximum Principles in Differential Equations, Printice-Hall, Englewood Cliffs, 1967.

[21] W. A. Strauss, Existence of solitary waves in higher dimensions, Comm. Math. Phys., 55 (1977), 144-162.

\author{
Department of Mathematics \\ FacUlty of Science and EngineERING \\ SAGA UNIVERSITY \\ SAGA 840, JAPAN
}

